\title{
Integer numbers and their ratios are key concepts in describing the interactions of atoms and molecules
}

\author{
Paul De Bièvre
}

Published online: 1 February 2011

(C) Springer-Verlag 2011

A general discussion of the redefinition of the mole, the SI unit for 'amount of substance', is now ongoing in ever broadening circles as part of a general overhaul of the Système International d'Unités, SI. Important for chemists are the unit mole (symbol mol) and the unit kilogram (symbol kg).

For several thousands of years, 'mass' was the property enabling measurement of "how much is there?" by using a comparative balance and the property of masses on the surface of the earth commonly called "weight" (concepts put in single quotes; double quotes used for quotations or to emphasize). Then, Isaac Newton (1642-1727) fundamentally changed our thinking by explaining that 'mass' was related to both 'gravitational attraction' and 'inertia'. Einstein (1879-1955) further showed that these are the same quantity. Quite different to this is the modern concept of 'amount of substance' mentioned in the SI but basically different from each of these. Equally important is the concept 'number of particles' (in generic language: 'number of entities'; 'entities' here must be specified), based on the property of 'numerosity' [1]. It is present in the mind of chemists making analytical measurements. 'Mass' was included in the International System of Quantities (ISQ) as a base quantity (Sect. 1.2 pp 104-105 in [2] and entry 1.6 in the Vocabulaire International de Métrologie-VIM [3]), and assigned the base unit kilogram. 'Numerosity' was not. Neither was 'number of entities'. This disregarded the discontinuous structure of matter.

In 1971, the Conférence Générale des Poids et Mesures (CGPM), on the proposal of the Comité International des Poids et Mesures (CIPM) and without a consultative committee on chemical measurements in existence (that

P. De Bièvre $(\square)$

Kasterlee, Belgium

e-mail: paul.de.bievre@skynet.be
Committee started to be operational in 1996 only), decided to define a unit "mole" as follows (see Sect. 2.1.1.6 p 115 in [2]):

The mole is the amount of substance of a system which contains as many elementary entities as there are atoms in $0.012 \mathrm{~kg}$ of carbon 12; its symbol is "mol."

When the mole is used, the elementary entities must be specified and may be atoms, molecules, electrons, other particles, or specified groups of such particles.

The mole is a base unit of the Systèmè International d'Unités.

The following addition was made in 1980 (p 115 in [2]):

In this definition it is understood that unbound atoms of carbon 12, at rest and in their ground state, are referred to.

Thus, the definition of the mole was made dependent on the definition of the unit for mass measurements though their corresponding base quantities were fundamentally independent (this is not unique in the SI). It is worthwhile to note that neither the CIPM nor the Bureau International des Poids et Mesures (BIPM) considered it necessary to have the measurement of chemical composition (a matter of 'number of entities') present in the CIPM and Consultative Committee structures of the SI, until about 1990 [4].

In recent years, various redefinitions of the mole have been under discussion (together with redefinitions of other SI base units) in specialized literature (Metrologia, Measurement, Chemistry International). These redefinitions are scheduled for decision by the CGPM in 2015. Hence, the Comité Consultatif pour la Quantité de Matière-Métrologie en Chimie (CCQM-MiC) to the CIPM had a brief discussion at its sixteenth session (April 2010) [5]. The minutes show "that there was agreement that, prior to the change, a more widespread understanding of the concepts 
and their acceptance within the chemical community must be achieved" (Sect. 7 p 9 in [5]). The President asked that the Delegates of the International Union of Pure and Applied Chemistry (IUPAC) to CCQM should "reflect a broad consensus in the chemical community." In its Recommendation Q 1 to the CIPM (p 40 in [5]), we read that CCQM:

\section{noting}

- that the level of awareness of the proposal to redefine the mole is low in the relevant community

- that support for the proposal to redefine the mole is not yet unanimous

recommends that any decision on redefining the mole and kilogram be deferred until

- full consideration be given to the interests of the chemical measurement community

- the BIPM, the National Measurement Institutes, and the other official representatives in the Consultative Committees increase their efforts to spread awareness of the proposals to the various scientific, industrial, and professional organizations, and seek their views at an early stage

states its preference for a redefinition of the mole, the SI unit for amount of substance, based on a fixed value of the Avogadro constant."

So far, discussions have occurred in a rather small, highlevel group of theoretical physicists who mainly discussed the subject in highly specialized literature for physicists and "metrologists." This is a regrettable situation since 'metrology' is the "science of measurement and its application" (entry 2.2 in [3]) and therefore applies to anybody making a measurement. It is the perception of this Editor as a chemist and on the basis of decades of active work in measurement (1961-1998) followed by many lectures and seminars on the five continents (1990-2010 and ongoing), on the introduction of metrological principles and terminology in measurements of chemical composition ("Metrology in Chemistry"-MiC), that the chemists have largely stayed away from discussing the opaque definition of the mole. Many teachers have problems attempting to explain the SI definition of the mole and of the concept 'amount of substance' to students and even to university staff. Chemists, especially the wet-analytical chemists, active in stoichiometry work involving dissolution, preparation of pure substances, drying precipitation, weighing, etc., were not involved in the present international debate too much, nor did they excessively involve themselves visibly, thus ignoring the issue at stake. Yet, it was "wet-analytical" chemists some 200 years ago (quoted by name below), who laid the foundation of stoichiometry in chemistry and developed the procedures and concepts leading to the modern chemical sciences and the methods of chemical analysis that make them possible. It seems an unanswered question why the actual quantity and unit they used, is not anchored in the SI. This may require the involvement of committee ISO/TC 12 [6] to conceive a new quantity.

\section{What is this issue of ACQUAL intended to bring?}

This issue of Accreditation and Quality Assurance (ACQUAL) is intended to open the debate much more than has been the case so far, e.g., to bring measurement of both small as well as large numbers into the SI.

\section{What is doubtful about the "mole"?}

The current definition of the mole tells us that one mole has a mass in gram the numerical value of which is equal to the corresponding relative atomic (or molecular) mass. A paper in this issue of ACQUAL discusses the "mole-concept compatibility condition" requiring Avogadro's number to be equal to the gram-to-dalton mass ratio, "a condition which must be kept" according to the author of the paper. The reason it is an essential condition is that it is a requirement for integrating atomic level measurements with measurements on the macroscopic scale using clearly connected units. This does not seem to be obeyed anymore in the redefinition recently proposed by the Comite Consultatif pour les Unités (CCU) [7], "thus requiring correction factors and creating potential confusion" according to this paper. This is a serious problem requiring much more thorough discussion than has occurred up to now in the chemical measurement community.

Several articles draw the attention to the desirability for a measurement and unit system that is quantized, using the concept of 'integer number'. The question is asked whether the Avogadro constant, as presently defined as a number per mol, i.e., $\mathrm{mol}^{-1}$, thus having the dimension $\mathbf{N}^{-1}$ (Sect. $1.3 \mathrm{p} 105$ [2]), and which is fit for the purpose of acting as a measurement unit for amount of substance, is fit for the purpose of measuring 'number of entities' or ratios of 'numbers of entities'. The latter is what analysts have so frequently in mind when measuring in the laboratory. Could it be that the Avogadro constant $N_{\mathrm{A}}$ has been conceived to enable definition of the quantity 'amount of substance', symbol $n$, "the quantity of which the presently defined mole is the unit," a seemingly circular definition?

\section{Counting (and measuring ratios of large numbers)- wouldn't that help?}

One study involves the "exceedingly simple" concept of a single entity (symbol "ent" as unit that requires no further 
definition). Would not the simple base quantity 'number of entities' give rise to an extremely simple "new" mole constituted by a 'number of (specified) entities', which is valid for all possible entities and which is de facto present in the mind of most chemists? That would lead to $1 \mathrm{~mol}=N_{\mathrm{avo}}$ (1 ent) where $N_{\text {avo }}$ would be Avogadro's number and ent the symbol for entity. Is there a historical basis for a quantity 'number of entities'? It is probably the oldest quantity there. The very earliest human records are about numbers of bags of grain, of numbers of soldiers, cattle, fruits, etc. Practical counting arithmetic probably predates the invention of writing. However, it is a recent insight that matter is constituted of discrete particles. It was suspected on the basis of careful logical argumentation as early as about 400 years BC by Demokritus (460-370 BC) and his predecessors. Much later, it was confirmed by the experimental work of $\mathrm{A}$. L. Lavoisier (1743-1793), J. L. Proust (1754-1826), J. Dalton (1766-1844), A. Avogadro (1776-1856), J. J. Berzelius (1779-1848), and W. Prout (1785-1850). J. B. Perrin (1870-1942) actually measured Avogadro's number experimentally and got the Nobel Prize in 1926 "for his work on the discontinuous structure of matter" [8]. Atoms and molecules are basic concepts created by the human mind and now an important part of our insight in nature.

That had far-reaching consequences.

The "discontinuous structure of matter" [8] enabled the study of matter in terms of the interaction of individual atoms and molecules, thus laying the foundation of what we now consider modern chemistry. Chemical phenomena could be traced to the particulate nature of matter: atoms and molecules. In other words, matter was confirmed to have the property of 'numerosity' [1]. This discovery was made by combining measurement results of mass ratios (measured by means of a balance) or ratios of gas volumes, with the concepts of relative atomic or relative molecular weight ("relative weight" in that context is also a ratio). The new insight led to thinking in numbers of atoms and molecules, which is of paramount importance as a(n integer) number of atoms constitute a molecule. Thinking in terms of numbers will expand further in the future with the development of biochemical and biological measurements:

- measurements in microbiology are a matter of measuring (counting) a 'number of entities' (e.g., specified bacterial cells);

- a 'number of active sites' on the surface of a protein molecule determines its role in any protein chemistry including disease fighting (upon invasion by bacteria).

It would enhance the status and authority of the SI in the chemical measurement community, if the measurement of 'numbers' would be (seen as) falling within its remit.

\section{What is 'amount of substance'?}

A large majority of chemists will reply: a 'number of atoms', or a 'number of molecules', i.e., in generic language, a 'number of entities'. That being the de facto perception since roughly two centuries, why is 'number of entities' not a base quantity in the SI? One would expect 'one entity' to be the natural unit on the atomic level for a measurement of a 'number of entities' (however large, up to the macroscopic level), each 'number of entities' being an exact multiple of 'one entity' (hence an integer number). That would correspond to a discrete, quantized unit such as 'one entity'. The intuitive thinking of the chemist does not seem to be reflected in the conceptual and terminological tools outlined in the SI. The value of a quantity, $Q$, can be expressed in that system as the product of a number or numerical value $\{Q\}$ and a unit $[Q]$ of the same quantity (Sect. 1.1 p 103 and Sect. 5.3.1 p 131 in [2]), i.e., $Q=\{Q\} \cdot[Q]$. In the case that $Q$ just is a 'number of entities', there is a problem as there is no unit for that quantity in the SI, and integer values are not a possibility, although the VIM recognizes the number one for that (entry 1.4 Note 3 in [3].

The CCQM in its sixteenth session [5] appears to have arrived at the same conclusion with respect to the desirability of changing the name "amount of substance". So has IUPAC in their motion to the CCU at its 2009 General Assembly in Glasgow [9] in which it requests explicitly that "the greatest effort should be made to change the name of the ISQ base quantity 'amount of substance' at the same time that a new definition of the mole is approved." Will changing the name change what some consider an error of concept?

To the best of our knowledge, this recommendation has not (yet?) been carried out. A suggestion to that effect was already made by proposing 'chemon' in 2000 [10]. The key concept in 'amount of substance' is 'amount'. For countable objects, number is the appropriate term. Should we not take inspiration from the fact that 'substance concentration', not 'amount-of-substance concentration', is used as 'measurand' (entry 2.1 in [3]) in clinical analytical measurements?

\section{Can a number be a unit?}

In other contributions in this issue of ACQUAL, it is discussed whether the number "one" can be a unit, as already proposed in 2004 [11], or whether a large number can be a unit that is a multiple of "one". But "one entity" can be a unit and a very useful one, so can a large 'number of entities' (a defined multiple of "one entity" on the macroscopic level). The 'number ratio' between large numbers of entities and one entity is of basic importance as scaling factor between the atomic and the macroscopic world [12]. In the mind of 
most chemists, it is the role the Avogadro number is fulfilling. The 'number-of-entities ratio' of quantities on the atomic level is exactly equal to the 'number-of-entities ratio' of the same quantities on the macroscopic level. Measuring the latter gives access to the former. Consequently, the question is anchored quite deeply in the minds of chemists, "Why don't we have the Avogadro number in the SI as the factor bridging the macroscopic and the atomic world?"

\section{What is the new SI?}

The debate on the redefinition of the mole is even more complex. A proposal has been tabled to redefine the mole based on the well known-and impressive-knowledge of the interrelationship of so-called fundamental constants. This approach is called the "fixed-constant" or "explicitconstant" approach and contrasts to the "fixed-unit" or "explicit-unit" approach. It is already incorporated in the draft of a revised Chap. 2 of the SI brochure, publicly available [7]. It is based on fixing the fundamental constants and making units dependent on them. Thus, the kilogram would be based on the Planck constant and become a "fixed Planck constant kilogram." More precisely, the mass of the unit kilogram would be such that the Planck constant $h=6.626068961 \times 10^{-34} \mathrm{~J} \mathrm{~s}$ [7]. In the present proposals for redefinition of the SI units, which would include the mole, the fixed-constant units would form what is already called the "new SI." Does not the 'fixed constant' approach turn units into variables and would they therefore not be made unsuitable to study small variations in the "fundamental constants"? Since 'comparability of measurement results' (entry 2.46 in [3]) requires metrological traceability to common references, usually units (entry 2.41 in [3]), said comparability requires "references" for the metrological traceability of measurement results including 'measured quantity values' (entry 2.10 in [3]) for fundamental constants. Nobody can predict whether, admittedly small, variations could not be uncovered in the future (discussions on this possibility are already going on among theoretical physicists in the specialized literature). Does making fundamental constants constant by definition not prevent future generations to study their (potential) variations? Also, a fixed or explicit constant definition of our measurement units based on these fixed constants, would in principle, have to be reviewed every time the Committee on Data Evaluation (CODATA) of the International Council of Scientific Unions (ICSU) reevaluates the values of the fundamental constants (including the "Avogadro constant") [7] presumably every 6-10 years. Does the measurement community approve a revision of the measurement units every $6-10$ years? It would be very difficult to explain to students in chemistry from primary school up to $\mathrm{PhD}$ candidates.

\section{A broad discussion is essential for chemists}

The "new SI" [7] is revolutionary. It will concern everybody working along "metrological" guidelines, and chemists must take care that their voices have an impact on any "new" system as it is likely to stay with them for decades.

Various other considerations are offered in this issue of ACQUAL. They have not yet been discussed thoroughly enough on a wide scale in the twenty-first century chemical measurement community, nor in the IUPAC Analytical Chemistry Division, nor in some other IUPAC Divisions, nor in CCQM. To stimulate these is the aim of ACQUAL. May the readers find that useful.

Comments and reactions are urgently invited, either as contributions to the Discussion Forum or in the form of a Letter to the Editor in this Journal. Let them be inspired by an intention to come to a transparent definition of the SI unit mole in the "new SI" [7], understood by the measurement community concerned.

The Editor wants to acknowledge (in)valuable constructive comments of many colleagues all over the world.

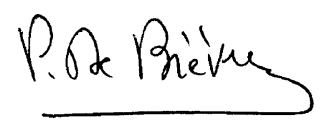

Paul De Bièvre

Editor-in-Chief

\section{References}

1. De Bièvre P (2007) Numerosity versus mass. Accred Qual Assur $12: 221-222$

2. Le Système international d'unités-The International System of Units SI, edition 8, ISBN 92-822-2213-6, BIPM, 2006, www.bipm.org/utils/common/pdf/si_brochure_8_en.pdf

3. BIPM, IEC, IFCC, ILAC, IUPAC, IUPAP, ISO, OIML (2008) The international vocabulary of metrology - basic and general concepts and associated terms (VIM), $3^{\text {rd }}$ edn. JCGM 200:2008, BIPM 2008, Paris-Sèvres, ISBN 92-822-2213-6 http://www.bipm.org/vim

4. Private communication from some CIPM members in 1992 to this editor

5. Report of the 16th session of CCQM, BIPM (2010) http://www. bipm.org/utils/common/pdf/CCQM16.pdf

6. ISO 80000-9 and 80000-10, www.iso.org, ISO/IEC directives, part 1, developed by ISO/TC 12, Quantities and units, International Organization for Standardization, ISO, Geneva, 2010

7. www.bipm.org/utils/common/pdf/CCU20.pdf

8. Perrin JB (1926) Discontinuous structure of matter, Nobel lecture 1926, http://nobelprize.org/nobel_prizes/physics/laureates/1926/ perrin-lecture.html

9. Lorimer J (2010) Chem Int 32:6-7

10. Dybkaer R (2000) Metrologia 37:301-304

11. Dybkaer R (2004) Metrologia 41:69

12. De Bièvre P (1990) Fresenius J Anal Chem 337:766-771 